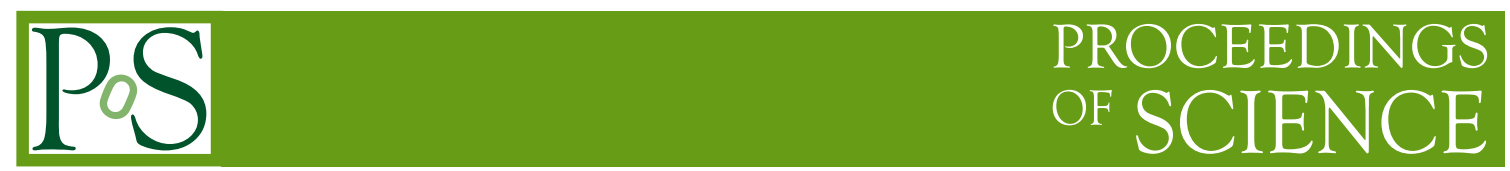

\title{
Status of MonteCarlo tools for SUSY and BSM Higgs physics
}

\section{Marco Zaro*}

Sorbonne Universités, UPMC Univ. Paris 06, UMR 7589, LPTHE, F-75005, Paris, France and

CNRS, UMR 7589, LPTHE, F-75005, Paris, France

E-mail: zaro@lpthe.jussieu.fr

In this talk, I will review the status of MonteCarlo tools for the simulation of BSM processes related to SUSY and BSM Higgs physics, focusing on those tools that include NLO QCD corrections matched to parton-shower.

Prospects for Charged Higgs Discovery at Colliders 3-6 October 2016

Uppsala, Sweden

${ }^{*}$ Speaker. 


\section{Introduction}

MonteCarlo event generators (MCs) are the workhorses for physics analyses at the LHC. Unweighted events generated by these tools can be passed through parton shower and detector simulations, bringing theoretical predictions as close as possible to the experimental measurements, without the need to apply corrections factors a posteriori that account for hadronization and detector effects. The description of the final state can be improved by including QCD effects, either via the explicit description of extra jet radiation, or by including NLO QCD corrections matched to parton shower simulations (or both). The inclusion of NLO QCD corrections makes it possible to have more reliable predictions for rates, shapes, and their theoretical uncertainties, together with a reduction of the latter with respect to LO simulations. Most of the modern NLO MCs strongly rely on automation, which makes it possible to hide the complexity of higher order calculations from the final user, and assures the correctness of calculations once building blocks are tested. While until few years ago the benefits of automations were restricted to processes in the Standard Model (SM), it is now possible to have NLO calculations from virtually any theory, the only necessary ingredient being the theory Lagrangian. Tools exist [1] that makes it possible to automatically extract the Feynman rules from the Lagrangian, including those Feynman rules which are necessary for the numerical evaluation of the one-loop matrix elements.

While for the SM case the benefits that one gets by including higher-order corrections are crystal-clear, one may wonder why one should bother in the case of BSM physics, which is still to be discovered. On the one hand, the inclusion of higher order corrections may significantly change the cross section, shifting (in some cases in quite a dramatic way) the exclusion contour. On the other hand, the smaller uncertainties that affect NLO simulations are reflected in more accurate, therefore more reliable, exclusion limits (see e.g. Ref. [2]).

In this talk I will review the status of MC simulations in BSM theories relevant for Higgs physics.

\section{MonteCarlos for the production of SUSY particles}

Possibly all production modes a pair of SUSY particles are available in NLO MCs, mostly implemented in the PowhegBox [3], and, more recently, also in MADGRAPH5_AMC@NLO [4]. They include the production of a sleptons pair [5] also including an extra jet [6], the production of a squark pair [7, 8], the production of a pair of stop quarks or of a pair of sgluons [9], the production of a pair of gluinos [10] and finally the production of a pair of electro-weakinos [11]. However, besides the case of slepton-pair production, no process featuring one or more jets is available at NLO accuracy. The reason for this is the following: consider the case of the production of a squark pair, $p p \rightarrow \tilde{q} \tilde{q}$. Among the real corrections belonging to the process $p p \rightarrow \tilde{q} \tilde{q} j$, one has also e.g. contributions where an intermediate gluino is produced, which sequentially decays into a squark and a jet. These contributions, which are actually LO contributions to a different process than the one considered (squark-gluino associated production), have to be removed, and techniques have been designed to remove them from the real corrections at a fully differential level [12]. These 
techniques which fall in the class of Diagram Removal (DR) or Diagram Subtraction (DS). Before briefly describing these techniques, I would like to mention that in the SM one has similar issues only for few processes, essentially related to single-top and $W$ production $[12,13,14]$, possibly in association with extra particles, such as a Higgs [15] or a $Z$ boson. Coming back to the techniques to remove resonant contributions, DR techniques are the simplest ones, as they just amount to remove the unwanted resonant diagrams from the amplitude (possibly retaining the interference between resonant and non-resonant diagrams). However, doing so, one might encounter issues due to non-gauge invariance when one moves away from the mass-shell of the resonant particle. DS techniques have been developed to deal with gauge-invariance issues. In these techniques the subtraction of resonant contributions is achieved by building local counterterms which are constructed from resonant diagrams evaluated on their mass-shell, and subtracted all-over the phase space by employing some momentum reshuffling. However, such a reshuffling can be performed in possibly infinite way, leading to ambiguities on what one defines as the subtracted cross section. Now, all these techniques are designed to deal with resonances that appear only in the real emissions. If one would start with one extra jet at the LO, the problem would appear also for Born and virtual amplitudes, and no technique exist to remove these extra contributions. This discussion is far from being purely of academic interest, as one may want, for example, to produce merged event samples at NLO $[16,17,18]$ to have a simultaneous NLO description of the 0 - and 1 -jet bin. However, this is currently not possible, except if one uses simplified models, i.e. models in which all particles which are not in the final state are decoupled from the theory. Future studies along these directions are certainly welcome.

\section{MonteCarlos for the simulation of neutral Higgs production in BSM theories}

In BSM theories such as SUSY or two-Higgs-doublet Models (2HDMs), the neutral Higgs states are dominantly produced in gluon-gluon fusion ( $\mathrm{ggF}$ ). Compared to the SM case, where the cross-section stems from the top quark and the bottom quark (the latter giving a minor contribution) circulating in the loop, in these BSM scenarios ggF can get extra contributions from extra heavy particles running in the loop, as well as from an enhanced coupling between the Higgs and bottom quarks. Being ggF a process that starts at one-loop already at LO, it cannot be tackled by standard automated NLO tools, rather ad-hoc and model-dependent implementations have to be produced. ggF production of a scalar and pseudo-scalar Higgs boson in the 2HDM or in the minimal-supersymmetric Standard Model (MSSM), have been implemented in the PowHEG framework [19] and in the MADGRAPH5_AMC@NLO one. In the latter case amplitudes from SUSHI [20] are used, and the resulting NLO+PS public code has been dubbed AMCSUSHI [21]. Studies done with these tools have shown that the effect of other particles running in the loop, such as squarks, cannot be neglected, and can have sizeable effects in both on the total rate and on the shape of the Higgs $p_{T}$ distribution. Studies have also appeared where the effects of the different treatment of resummation, as well as of different choices for the resummation scales [22]. Recently, SUSHI has been extended by including the amplitudes for scenarios beyond the MSSM in particular for the NMSSM [23]. Both POWHEG and AMC @ NLO matching are available, as well as the possibility to perform analytic resummation for the Higgs transverse momentum (MORE-SUSHI [24]). 
Besides ggF, another Higgs production mechanism which is relevant in BSM scenarios, particularly in those where the bottom-Higgs coupling is enhanced, is $b \bar{b} H$ associated production. Since searches for this channel strongly rely on tagging one or more $b$ jets, an accurate description of these observables is necessary, and it can be achieved by using the so-called four-flavour scheme (4FS), which retains bottom mass effects. In Ref [25] the first calculation of $b \bar{b} H$ in the 4FS at NLO+PS accuracy has been performed in the MADGRAPH5_AMC@NLO framework, together with a thorough comparison against the 5FS, which shows the limit of the latter scheme in particular when observables related to the bottom quarks are studied. A POWHEG implementation of the same process has also appeared [26] and, in the context of the LHC Higgs Cross-Section Working Group (LHCHXSWG), a comparison of the various tools has been performed [27].

\section{MonteCarlos for the simulation of charged Higgs production}

Charged Higgs boson appear in many extensions of the SM, such as the 2HDM, the MSSM as well as more exotic models. For what concerns the production of charged Higgs bosons in the 2HDM and in the MSSM, recent results [28, 29] have been covered in Maria Ubiali's talk in this conference [30]. Therefore, in my talk, I will focus on a more exotic case, the Georgi-Machacek (GM) model [31, 32], where one has several charged Higgs bosons in the spectrum, including also two doubly charged ones. Unlike in the case of 2HDM and the MSSM, where one adds extra SU(2) doublets to the theory, in GM models one adds, besides the "standard" Higgs doublet responsible for the fermion masses, two triplets (one real and one complex). After symmetry breaking, singly and doubly charged Higgs bosons appear in the particle spectrum, as well as a non-vanishing level coupling between two vector bosons and a charged Higgs, a feature which is absent in models where only doublets are added. This makes Vector-Boson Fusion (VBF) the dominant production mode for charged Higgs. The inclusive cross section for VBF production has been computed since some time ago at NNLO QCD accuracy [33, 34, 35, 36]. However only more recently, thanks to NLO automation, it has been possible to simulate Charged Higgs production in GM models in a fully-differential manner at NLO+PS accuracy [37] ${ }^{1}$. This is not restricted to the dominant production mode, VBF, but also for the subleading production channel of a charged Higgs boson: $H^{ \pm} V$ associated production and double Higgs production. This is another example of the benefits of NLO automation, which now makes it possible to compute NLO QCD corrections to any process also in exotic theories, almost without efforts.

\section{Conclusions}

Searches for new physics at the LHC need reliable fully-differential predictions. NLO MonteCarlos make it possible to profit of the benefits of NLO calculations in fully-differential analyses, and, while only few years ago they were mostly restricted to the case of SM processes, the growing level of automation of these tools has led to having this possibility also for BSM cases, although with some limitations. One of such limitations is related to processes which have resonances that appear in the real emission corrections, and to the fact that it is not known yet how to treat these resonances in processes which feature jets at the LO. Another limitation of automatic tools concerns

\footnotetext{
${ }^{1}$ The model files are available online, https://feynrules.irmp.ucl.ac.be/wiki/NLOModels
} 
processes which are loop-induced, as it is the case for Higgs production in $\mathrm{ggF}$, the dominant Higgs production channel in the SM and in most of its extensions. For these processes, a model-specific implementation of the relevant amplitudes is necessary. Anyway, for processes which do belong to these two cases, automatic tools have made possible to do calculations which would have needed years of work each, almost without any effort.

\section{Acknowledgements}

I would like to thank the organizers for the kind invitation to give this talk at such a nice conference. My work is supported by the European Union's Horizon 2020 research and innovation programme under the Marie Sklodovska-Curie grant agreement No 660171 and in part by the ILP LABEX (ANR-10-LABX-63), in turn supported by French state funds managed by the ANR within the "Investissements d'Avenir" programme under reference ANR-11-IDEX-0004-02.

\section{References}

[1] C. Degrande, Comput. Phys. Commun. 197 (2015) 239 doi:10.1016/j.cpc.2015.08.015 [arXiv:1406.3030 [hep-ph]].

[2] C. Arina et al., JHEP 1611 (2016) 111 doi:10.1007/JHEP11(2016)111 [arXiv:1605.09242 [hep-ph]].

[3] S. Alioli, P. Nason, C. Oleari and E. Re, JHEP 1006 (2010) 043 doi:10.1007/JHEP06(2010)043 [arXiv:1002.2581 [hep-ph]].

[4] J. Alwall et al., JHEP 1407 (2014) 079 doi:10.1007/JHEP07(2014)079 [arXiv:1405.0301 [hep-ph]].

[5] B. Jager, A. von Manteuffel and S. Thier, JHEP 1210 (2012) 130 doi:10.1007/JHEP10(2012)130 [arXiv:1208.2953 [hep-ph]].

[6] B. Jager, A. von Manteuffel and S. Thier, JHEP 1502 (2015) 041 doi:10.1007/JHEP02(2015)041 [arXiv:1410.3802 [hep-ph]].

[7] R. Gavin, C. Hangst, M. Krämer, M. Mühlleitner, M. Pellen, E. Popenda and M. Spira, JHEP 1310 (2013) 187 doi:10.1007/JHEP10(2013)187 [arXiv:1305.4061 [hep-ph]].

[8] R. Gavin, C. Hangst, M. Krämer, M. Mühlleitner, M. Pellen, E. Popenda and M. Spira, Eur. Phys. J. C 75 (2015) no.1, 29 doi:10.1140/epjc/s10052-014-3243-2 [arXiv:1407.7971 [hep-ph]].

[9] C. Degrande, B. Fuks, V. Hirschi, J. Proudom and H. S. Shao, Phys. Rev. D 91 (2015) no.9, 094005 doi:10.1103/PhysRevD.91.094005 [arXiv:1412.5589 [hep-ph]].

[10] C. Degrande, B. Fuks, V. Hirschi, J. Proudom and H. S. Shao, Phys. Lett. B 755 (2016) 82 doi:10.1016/j.physletb.2016.01.067 [arXiv:1510.00391 [hep-ph]].

[11] J. Baglio, B. Jager and M. Kesenheimer, JHEP 1607 (2016) 083 doi:10.1007/JHEP07(2016)083 [arXiv:1605.06509 [hep-ph]].

[12] S. Frixione, E. Laenen, P. Motylinski, B. R. Webber and C. D. White, JHEP 0807 (2008) 029 doi:10.1088/1126-6708/2008/07/029 [arXiv:0805.3067 [hep-ph]].

[13] C. D. White, S. Frixione, E. Laenen and F. Maltoni, JHEP 0911 (2009) 074 doi:10.1088/1126-6708/2009/11/074 [arXiv:0908.0631 [hep-ph]].

[14] E. Re, Eur. Phys. J. C 71 (2011) 1547 doi:10.1140/epjc/s10052-011-1547-z [arXiv:1009.2450 [hep-ph]]. 
[15] F. Demartin, B. Maier, F. Maltoni, K. Mawatari and M. Zaro, arXiv:1607.05862 [hep-ph].

[16] R. Frederix and S. Frixione, JHEP 1212 (2012) 061 doi:10.1007/JHEP12(2012)061 [arXiv:1209.6215 [hep-ph]

[17] L. Lönnblad and S. Prestel, JHEP 1303 (2013) 166 doi:10.1007/JHEP03(2013)166 [arXiv:1211.7278 [hep-ph]].

[18] M. Schonherr, S. Hoeche, F. Krauss and F. Siegert, PoS ICHEP 2012 (2013) 314 [arXiv:1212.0386 [hep-ph]].

[19] E. Bagnaschi, G. Degrassi, P. Slavich and A. Vicini, JHEP 1202 (2012) 088 doi:10.1007/JHEP02(2012)088 [arXiv:1111.2854 [hep-ph]].

[20] E. Bagnaschi, R. V. Harlander, S. Liebler, H. Mantler, P. Slavich and A. Vicini, JHEP 1406 (2014) 167 doi:10.1007/JHEP06(2014)167 [arXiv:1404.0327 [hep-ph]].

[21] H. Mantler and M. Wiesemann, Eur. Phys. J. C 75 (2015) no.6, 257 doi:10.1140/epjc/s10052-015-3462-1 [arXiv:1504.06625 [hep-ph]].

[22] E. Bagnaschi, R. V. Harlander, H. Mantler, A. Vicini and M. Wiesemann, JHEP 1601 (2016) 090 doi:10.1007/JHEP01(2016)090 [arXiv:1510.08850 [hep-ph]].

[23] S. Liebler, H. Mantler and M. Wiesemann, arXiv:1608.02949 [hep-ph].

[24] R. V. Harlander, H. Mantler and M. Wiesemann, JHEP 1411 (2014) 116 doi:10.1007/JHEP11(2014)116 [arXiv:1409.0531 [hep-ph]].

[25] M. Wiesemann, R. Frederix, S. Frixione, V. Hirschi, F. Maltoni and P. Torrielli, JHEP 1502 (2015) 132 doi:10.1007/JHEP02(2015)132 [arXiv:1409.5301 [hep-ph]].

[26] B. Jager, L. Reina and D. Wackeroth, Phys. Rev. D 93 (2016) no.1, 014030 doi:10.1103/PhysRevD.93.014030 [arXiv:1509.05843 [hep-ph]].

[27] D. de Florian et al. [LHC Higgs Cross Section Working Group Collaboration], arXiv:1610.07922 [hep-ph].

[28] C. Degrande, M. Ubiali, M. Wiesemann and M. Zaro, JHEP 1510 (2015) 145 doi:10.1007/JHEP10(2015)145 [arXiv:1507.02549 [hep-ph]].

[29] C. Degrande, R. Frederix, V. Hirschi, M. Ubiali, M. Wiesemann and M. Zaro, arXiv:1607.05291 [hep-ph].

[30] M. Ubiali, these proceedings.

[31] H. Georgi and M. Machacek, Nucl. Phys. B 262 (1985) 463. doi:10.1016/0550-3213(85)90325-6

[32] M. S. Chanowitz and M. Golden, Phys. Lett. 165B (1985) 105. doi:10.1016/0370-2693(85)90700-2

[33] P. Bolzoni, F. Maltoni, S. O. Moch and M. Zaro, Phys. Rev. Lett. 105 (2010) 011801 doi:10.1103/PhysRevLett.105.011801 [arXiv:1003.4451 [hep-ph]].

[34] M. Zaro, P. Bolzoni, F. Maltoni and S. O. Moch, PoS CHARGED 2010 (2010) 028 [arXiv:1012.1806 [hep-ph]].

[35] P. Bolzoni, F. Maltoni, S. O. Moch and M. Zaro, Phys. Rev. D 85 (2012) 035002 doi:10.1103/PhysRevD.85.035002 [arXiv:1109.3717 [hep-ph]].

[36] M. Zaro and H. Logan, LHCHXSWG-2015-001.

[37] C. Degrande, K. Hartling, H. E. Logan, A. D. Peterson and M. Zaro, Phys. Rev. D 93 (2016) no.3, 035004 doi:10.1103/PhysRevD.93.035004 [arXiv:1512.01243 [hep-ph]]. 